\title{
SCATTERING OF LIGHT BY CHARGED COLLOIDAL PARTICLES IN SALT SOLUTIONS
}

\author{
A. Vrij and J. Th. G. Overbeek \\ van't Hoff Laboratory, University of Utrecht, the Netherlands \\ Received September 1, 1961
}

\begin{abstract}
In the interpretation of light scattering by colloidal electrolytes in salt solutions the interaction between the colloidal particles and the low molecular weight ions has to be taken into account. When fluctuation theory is applied for the derivation of a light-scattering equation, nonelectroneutral fluctuations may be neglected in most cases. The total light scattering can be split into three contributions, one due to density fluctuations, one due to concentration fluctuations in the low molecular weight components, and one due to the colloidal particles. In the last-named contribution the (usually negative) adsorption of the low molecular weight salts by the colloid is included. This can be taken into account in good approximation by using in the light-scattering equations the refractive index increment at constant chemical potential and not at constant concentrations of the other components of the system. This quantity can be measured directly in membrane equilibria or it can be calculated from concentration differences in a membrane equilibrium combined with the more usual refractive index increments at constant concentrations.

The theoretical treatment is confirmed by measurements of light scattering and membrane equilibria with half-neutralized polymethacrylic acid in $0.1 M$ sodium halide solutions and in a few other salts. The correction on the molecular weight varies from $10 \%$ in $\mathrm{NaF}$ to $25 \%$ in $\mathrm{NaI}$ and amounts even to $45 \%$ in 0.01 $M\left(\mathrm{NH}_{4}\right)_{6} \mathrm{Mo}_{7} \mathrm{O}_{24}$.
\end{abstract}

\section{INTRODUCTION}

In his theory of light scattering by colloidal solutions Debye (1) derived the following equation which is valid for a two-component system (colloid and solvent)

$$
\frac{H g}{\tau-\tau_{0}}=\frac{1}{R T} \frac{d \Pi}{d g} .
$$

Here $g$ is the concentration of the colloid in $\mathrm{g} . / \mathrm{cm}^{3}, \Pi$ is the osmotic pressure against the solvent, $R$ is the gas constant, $T$ is the absolute temperature, and $\tau$ and $\tau_{0}$ are the turbidities of solution and solvent, respectively. The factor $H$ has the value

$$
H=\frac{32 \pi^{3} n^{2}(d n / d g)^{2}}{3 \lambda_{0}{ }^{4} N_{A v}},
$$


where $n$ is the refractive index of the solution, $\lambda_{0}$ is the wavelength in vacuum of the light used for the experiment, and $N_{A v}$. is Avogadro's number.

With the following expression for the osmotic pressure

$$
\frac{\Pi}{R T}=\frac{g}{M}+B g^{2}
$$

Eq. [1] can be transformed into

$$
\frac{H g}{\tau-\tau_{0}}=\frac{1}{M}+2 B g .
$$

Here $M$ is the molecular weight of the colloid and $B$ is the second virial coefficient.

The above equations which have been derived from fluctuation theory are not wholly applicable to the case of charged colloidal particles in a salt solution. This is a three-component system where the fluctuations of colloid and salt are interdependent. Zernike (2) was the first to give a rigorous treatment of the light scattering in multicomponent systems. This treatment has been extended by Brinkman and Hermans (3), Kirkwood and Goldberg (4), Stockmayer (5), Shogenji (6), and recently by Ooi (7) and by Casassa and Eisenberg (8). In these theories suitable generalizations are derived for the quantities $H$ (containing the refractive index increments) and $d \Pi / d g$ (related to the free energy of the system). In electrolytic solutions, however, it is hard to find accurate explicit expressions for the free energy because activity coefficients are so hard to calculate.

Mysels et al. $(9,10)$ and Prins and Hermans (11) in studying light scattering by soap micelles tried to avoid activity coefficients by ascribing an "effective charge" to the micelles and further assuming ideal behavior for the solutions. This model is not satisfactory in so far as it is not evident whether the introduction of an effective charge takes the deviations from ideality sufficiently into account. We shall return to this point in a later paper. See also reference 12, chapter 5. Recently Stigter (13) has employed statistical thermodynamic relations to express the turbidity in terms of molecular parameters, i.e., size and surface charge of the colloidal particles and the ionic strength of the solution.

The theory faces another difficulty in the long-range nature of electrostatic interactions. If the ionic strength of the solution is very small $\left(<10^{-3} \mathrm{M}\right)$, the range of the interaction between charged particles becomes comparable with the wavelength of visible light. In this case interference effects occur (14). These cannot be described by fluctuation theory in its usual form, which assumes the fluctuations in neighboring volume-elements to be independent.

In this paper light scattering by charged particles in salt solutions will be treated by fluctuation theory. Only electroneutral fluctuations will be taken into account. The charge of the colloidal particles attracts counterions 
and pushes co-ions away, thus causing a negative adsorption of added salts. This negative adsorption fluctuates as a whole with the particles, so that one may say that the particle plus its complete surroundings is the scattering unit. The amount of negative adsorption can be determined exactly in a membrane equilibrium (Donnan equilibrium) between the colloid solution and a solution containing only its small molecular weight components.

\section{Fluctuation Theory}

The scattering solution, which may be a multicomponent system, is divided into volume elements $\Delta V$. The refractive index in such a volume element will fluctuate around a mean value owing to the Brownian motion of the particles.

Fluctuation theory may be applied if the following conditions are satisfied:

a. In order to keep phase differences negligible, the volume-elements $\Delta V$ should be small with respect to the wavelength of the light used.

$b$. However, the volume-elements should be large enough to make concentration fluctuations in a given volume-element independent of those in neighboring volume-elements (15).

If both these conditions are satisfied, the volume-elements may be considered as radiating dipoles the amplitudes of which fluctuate independently. Consequently the total scattering of the solution is equal to the sum of the contributions of all volume-elements. It may be written as follows $(2,3)$ :

$$
\tau=\frac{32 \pi^{3} \bar{n}^{2} V \overline{(\Delta n)^{2}}}{3 \lambda_{0}{ }^{4}}
$$

Here $\Delta n=n-\bar{n} ; n$ and $\bar{n}$ are the refractive index and the mean refractive index, respectively, in a volume $V ; \tau$ is the turbidity; $\lambda_{0}$ is the wavelength in vacuo.

We consider a scattering volume $V$, with a volume element $\Delta V$ in the shape of a slice with thickness $d$ (Fig. 1).

The maximum phase difference of light rays (with wavelength $\lambda_{m}$ in the medium) scattered under an angle $\theta$ by the volume element is equal to $2 d(\sin (\theta / 2)) / \lambda_{m}$. Hence to satisfy condition $(a)$ this quantity should be small with respect to unity.

In order to satisfy condition (b) the size of the particles and the range of the intermolecular forces ( $\operatorname{say} r_{0}$ ) should be small with respect to the thickness $d$ of the volume element $\left(r_{0} \ll d\right)$. For short-range forces and visible light both conditions can be satisfied for all scattering angles. For long-range forces, however, it is necessary to measure the scattering under such a small angle that $2 r_{0} \sin (\theta / 2) / \lambda_{m}$ be small with respect to unity. In order to find the scattering at larger angles, the correlations between different volume 


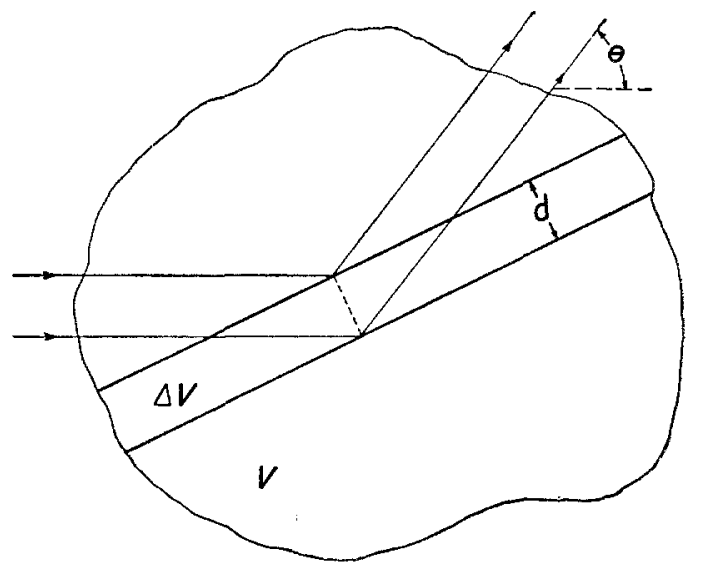

Fig. 1. Light scattered by the slice $\Delta V$ has a maximum phase difference $\left(2 d / \lambda_{m}\right) \sin (\theta / 2)$.

elements have to be taken into account, but extrapolation to a scattering angle $\theta=0$ restores the validity of $\mathrm{Eq}$. [5].

\section{Charged Particles}

In systems containing charged particles such as proteins, polyelectrolytes, and simple electrolytes, the range of the electrostatic forces is given by the thickness, $1 / K$, of the Debye-Hückel ionic atmosphere, where

$$
\frac{1}{K}=\sqrt{\frac{D k T}{4 \pi \sum_{i} e_{i}^{2} c_{i}}},
$$

and $D$ is the dielectric constant of the medium, $k$ the Boltzmann constant, $e_{i}$ the ionic charge, and $c_{i}$ the average number of ions of type $i$ per unit volume. Beyond a distance of a few times $1 / K$ from a charged particle systematic concentration differences are negligible. Hermans (15) has shown that in volume-elements whose linear dimensions are larger than $1 / K$, nonelectroneutral fluctuations may be neglected as compared to neutral fluctuations. Consequently, the fluctuation theory may be applied to systems containing charge particles if

$$
\frac{2 \sin \theta / 2}{K \lambda_{m}} \ll 1
$$

In order to keep deviations below 1\% the left-hand side of expression [7] should be smaller than 0.05 . See reference 15 . For visible light this condition obtains for all angles, if $K>1 /(100 \mathrm{~A}$.) or in aqueous solutions when the ionic strength is more than $10^{-3}$ molar. We shall restrict our considerations to cases where Eq. [7] is satisfied. 
Derivation of a Light-Scattering Equation for a Charged Colloid in a Salt Solution

In a system of $m+1$ components $(i=0,1,2 \cdots m)$ the mean square of the fluctuation in the refractive index $\overline{(\Delta n)^{2}}$ (see Eq. [5]) can be written:

$$
\overline{\Delta n^{2}}=\sum_{i, k=0}^{m} n_{i} n_{k} \overline{\Delta N_{i} \Delta N_{k}}=k T \sum_{i, k=0}^{m} n_{i} n_{k}\left(\partial \bar{N}_{i} / \partial \mu_{k}\right)_{\mu V T},
$$

where $n_{i}=\left(\partial n / \partial N_{i}\right)_{T V N} ; N_{i}$ is the number of particles of type $i$ in volume $V ; \Delta N_{i}=N_{i}-\bar{N}_{i} ; \mu_{k}$ is the chemical potential of component $k$. The indices $N, \mu$ mean that all $N_{j}, \mu_{j}$, except $N_{i}, \mu_{i}$ are kept constant in the differentiation with respect to $N_{i}$ or $\mu_{i}$, respectively. In the following discussion the index $T$ will be omitted for the sake of simplicity.

The first equality in Eq. [8] is self-evident. The second one is based on the relation

$$
\overline{\Delta N_{i} \Delta N_{k}}=k T\left(\partial \bar{N}_{i} / \partial \mu_{k}\right)_{\mu V}=k T\left(\partial \bar{N}_{k} / \partial \mu_{i}\right)_{\mu V},
$$

which was obtained by Zernike (2) using Gibbs' grand canonical ensemble.

Writing

$$
\Xi=\sum_{l, \underline{\underline{N}}} \exp \frac{\underline{\underline{N}}}{\underline{\underline{\underline{\mu}}}-E_{l}} \frac{E_{l}}{k T}
$$

for the sum of states in the grand ensemble (16), where $l$ indicates the different energy levels of the system, $\underset{\underline{N}}{\underline{n}}$ the different sets of $N_{0}, N_{1} \cdots N_{m}$, and $\stackrel{\underline{N}}{\underline{\mu}}=\sum_{i=0}^{m} N_{i} \mu_{i}$, we find the average value of $N_{j}$ from

$$
\bar{N}_{i} \Xi=\sum_{l, \underline{\underline{N}}} N_{i} \exp \frac{\underline{\underline{N}} \cdot \underline{\underline{\mu}}-E_{l}}{k T} .
$$

Differentiation with respect to $\mu_{k}$ then leads to the desired relation [9].

$$
\frac{\partial \bar{N}_{i}}{\partial \mu_{k}} \Xi+\bar{N}_{i} \sum_{l \cdot \underline{\underline{\underline{N}}}} \frac{N_{k}}{k T} \exp \stackrel{\underline{N} \cdot \underline{\underline{\underline{\mu}}}-E_{l}}{k T}=\sum_{l, \underline{\underline{\underline{N}}}} \frac{N_{i} N_{k}}{k T} \exp \frac{\underline{\underline{N}} \cdot \underline{\underline{\underline{\mu}}}-E_{l}}{k T}
$$

or

$$
\frac{\partial \bar{N}_{j}}{\partial \mu_{k}}=-\frac{\bar{N}_{i} \bar{N}_{k}}{k T}+\frac{\overline{N_{i} N_{k}}}{k T}=\frac{\overline{\left(N_{i}-\bar{N}_{j}\right)\left(N_{k}-\bar{N}_{k}\right)}}{k T}=\frac{\overline{\Delta N_{j} \Delta N_{k}}}{k T} .
$$

Before inserting Eq. [8] into the light-scattering Eq. [5] it is desirable to apply some transformation. In the first place the condition $V=$ constant will be eliminated from the differential quotients $n_{i}=\left(\partial n / \partial N_{i}\right)_{V N}$. Further, the total light scattering will be split into three contributions, one containing the density fluctuations, mainly from the solvent (component 0 ), one containing the concentration fluctuations of the colloid with an equivalent amount of counterions (component 1) including the contribu- 
tion from the positive or negative adsorption of the low molecular weight salts and other solutes (components $2 \cdots m$ ) to the colloid, and one derived from the concentration fluctuations of the remainder of the components $2 \cdots m$. Ooi $(7)$ has given such a derivation for a neutral polymer in a mixture of solvents. Casassa and Eisenberg (8) developed the theory for a polyelectrolyte with salt. Independently an analogous derivation was given by one of us (12) for the ionic case.

In Appendix I Eq. [12] is derived from Eq. [5] and Eq. [8].

$$
\frac{\tau}{A}=\left[V^{2}{n_{\nabla}}^{2}-\phi^{2}\right]_{\kappa}+\frac{\left[\sum_{i=1}^{m} \gamma_{i}\left(\frac{\partial c_{i}}{\partial c_{1}}\right)_{\mu_{s}}\right]^{2}}{\left(\partial \Pi / \partial c_{1}\right)_{\mu_{s}}} c_{1}+\sum_{i, k=2}^{m} \gamma_{i} \gamma_{k}\left(\partial c_{i} / \partial \mu_{k}\right)_{\mu_{s^{\prime}} c_{1}} \text {. }
$$

In this equation

$$
\begin{array}{rlr}
A & =32 \pi^{3} \bar{n}^{2} k T / 3 \lambda_{0}{ }^{4} ; & \\
n_{V} & =(\partial n / \partial V)_{N} ; & \\
\phi & =\sum_{i=1}^{m} \gamma_{i} c_{i} ; & \\
\gamma_{i} & =\left(\partial n / \partial c_{i}\right)_{P, e} ; \\
c_{i} & =\bar{N}_{i} / V ; \\
\kappa & =-(\partial V / V \partial P)_{N} ; \\
P= & \text { pressure; } \\
\Pi I= & \text { osmotic (Donnan) pressure difference between the solution and } \\
& \text { a solution in equilibrium with it but containing only the low } \\
& \text { molecular weight components. }
\end{array}
$$

The index $\mu_{s},\left(\mu_{s}{ }^{\prime}\right)$ means that the chemical potentials of all the solutes (all solutes except the one used in the differentiation) are kept constant. The first term of Eq. [12] is due to density fluctuations, the second term to the colloid including the contribution from the positive or negative adsorption of the noncolloidal solutes, and the third one to the concentration fluctuations of the remainder of the noncolloidal solutes. For a salt solution without colloid (components $0,2, \cdots m$ ) one obtains

$$
\frac{\tau^{*}}{A}=\left[V n_{V}^{* 2}-\phi^{* 2}\right] \kappa^{*}+\sum_{i, k=2}^{m} \gamma_{i}^{*} \gamma_{k}^{*}\left(\partial c_{i} / \partial \mu_{k}\right)_{\mu_{s^{\prime}}, c_{1}=0}
$$

We have labeled the variables here with an asterisk.

It is shown by a numerical argument in Appendix II that $\tau^{*} / A$ can be put equal to the corresponding unstarred terms of Eq. [12] in very good approximation:

$$
\frac{\tau^{*}}{A} \simeq\left[V n_{V}^{2}-\phi^{2}\right]_{\kappa}+\sum_{i, k=2}^{m} \gamma_{i} \gamma_{k}\left(\partial c_{i} / \partial \mu_{k}\right)_{\mu_{s^{\prime}, c_{1}}}
$$


and consequently

$$
\frac{\tau-\tau^{*}}{A}=\frac{\left[\sum_{i=1}^{m} \gamma_{i}\left(\partial c_{i} / \partial c_{1}\right)_{\mu_{s}}\right]^{2}}{\left(\partial \Pi / \partial c_{1}\right) \mu_{s}} c_{1},
$$

where $\tau^{*}$ is the turbidity of the salt solution without colloid in Donnan equilibrium with the solution of turbidity $\tau$.

The term $\Sigma \gamma_{i}\left(\partial c_{i} / \partial c_{1}\right)_{\mu_{s}}$ can be treated in two different ways.

$a$. The refractive index increments $\gamma_{i}=\left(\partial n / \partial c_{i}\right)_{p c}$ can be measured directly at normal pressure, and $\left(\partial c_{i} / \partial c_{1}\right)_{\mu_{s}}$, being the adsorption of component $i$ on the colloid, can be determined in a Donnan membrane equilibrium with an outside solution with chemical potentials $\mu_{s}$ and an inside solution with colloid concentration $c_{1}$ (or it may be calculated in special cases).

The determination of $\left(\partial c_{i} / \partial c_{1}\right) \mu_{\mathrm{s}}$ should strictly be carried out at the pressure of the Donnan cell, but the relative compressibility correction is of the order of $k c$ $\left(\partial \Pi / d c_{1}\right)_{\mu_{3}} /\left(\partial c_{i} / \partial c_{1}\right)_{\mu_{\mathrm{s}}}<1 / M_{\text {colloid }}$ and is thus completely negligible.

$b$. It is also possible to determine the sum $\Sigma \gamma_{i}\left(\partial c_{i} / \partial c_{1}\right)_{\mu_{\varepsilon}}$ directly by allowing the Donnan equilibrium to be established between two solutions with a difference in concentration $\left(\Delta c_{1}\right)$ of the colloid, separating the solutions and measuring the difference in refractive index $(\Delta n)$. Then $\Delta n / \Delta c_{1}$ is again with a relative error of the order $\kappa c\left(\partial \Pi / / d c_{1}\right) /\left(\partial c_{i} / \partial c_{1}\right)$ equal to the abovementioned sum.

In Ooi's analysis $(7)$ the term $\Sigma \gamma_{i}\left(\partial c_{i} / \partial c_{1}\right)_{\mu_{s}}$ is replaced by $\left(\partial n / \partial c_{1}\right)_{\mu_{s}}$, the value of $\tau^{*}$ being also slightly changed. In $\left(\partial n / \partial c_{1}\right)_{\mu_{s}}$ the increments of the refractive index and of the concentration $c_{1}$ should both be measured at the Donnan pressure. The difference, however, with $\Delta n / \Delta c_{1}$ as defined above and with $\Sigma \gamma_{i}\left(\partial c_{2} / \partial c_{1}\right)_{\mu_{s}}$ is again of the order of $1 / M_{\text {colloid }}$.

When concentrations by weight are introduced, $g_{i}=c_{i} M_{i} / N_{A v}$. , where $M_{i}$ is the molecular weight of component $i, \mathrm{Eq}$. [20] can be transformed into

$$
\frac{H^{\prime} g_{1}}{\tau-\tau^{*}}=\frac{1}{R T}\left(\frac{\partial \Pi}{\partial g_{1}}\right)_{\mu_{s}},
$$

where

$$
H^{\prime}=\frac{32 \pi^{3} \bar{n}^{2}}{3 \lambda_{0}^{4} N_{A v}}\left(\frac{\partial n}{\partial g_{1}}\right)_{\text {Donnan }}^{2}
$$

and $\left(\partial n / \partial g_{1}\right)_{\text {Donnan }}$ is the refractive index increment measured between the two solutions of a Donnan equilibrium, but brought to the same pressure.

The Donnan pressure II may be expanded into a power series in $g_{1}$ $(17,18)$

$$
\Pi / R T=g_{1} / M_{1}+B\left(\mu_{s}\right) g_{1}{ }^{2}+D\left(\mu_{s}\right) g_{1}{ }^{3}+\cdots
$$


Introducing this expression into Eq. [21] leads to

$$
\frac{H^{\prime} g_{1}}{\tau-\tau^{*}}=\frac{1}{M_{1}}+2 B\left(\mu_{s}\right) g_{1}+\text { etc. }
$$

an expression differing only from the traditional expression [4] in that $\tau^{*}$ now represents the scattering by the mixture of solvent(s) and low molecular weight salts and in that $H^{\prime}$ contains a refractive index increment at constant chemical potential rather than at constant concentration.

In an actual dilution series the concentration of the salts rather than their chemical potential will be kept constant. This implies that for each concentration of the colloid the chemical potentials $\mu_{s}$ will have slightly different values. This affects $H^{\prime}, \tau^{*}$, and $B$. The influence on $H^{\prime}$ is due to higher order effects such as $\left(\partial^{2} n / \partial g_{i} \partial g_{1}\right)$ and $\left(\partial^{2} g_{i} / \partial g_{1}{ }^{2}\right)$ and may be neglected.

It has been mentioned in Appendix II that the influence on $\tau^{*}$ is small compared to the difference between $\tau^{*}$ and $\tau^{0 *}$ and may also be neglected. The second virial coefficient finally may be written

$$
B\left(\mu_{s}\right)=B\left(\mu_{s}^{0}\right)+\frac{\partial B}{\partial \mu_{s}}\left(\frac{\partial \mu_{s}}{\partial g_{1}}\right)_{g} g_{1}+\cdots
$$

and the correction term therefore would affect only higher terms than the ones written in Eq. [24].

\section{True and Apparent Molecular Weight and Second Virial Coefficient}

The use of Eq. [24] allows the calculation of the true molecular weight $M_{1}$ and the true second virial coefficient $B$ from turbidity measurements, provided $\left(\partial n / \partial g_{1}\right)$ has been determined with a correct procedure, i.e., using a membrane equilibrium. If, however, a refractive index increment at constant concentrations ( $\left.\partial n / \partial g_{1}\right)_{g_{2} \cdots g_{m}}$ is substituted in expression [22] for $H^{\prime}$, then an incorrect or apparent molecular weight $M_{1}^{*}$ and second virial coefficient $B^{*}$ are found. If only one low molecular weight salt is present (component 2), the relation between $M_{1}$ and $M_{1}^{*}$ is

$$
M_{1}^{*}=M_{1}\left[1+\frac{\left(\partial n / \partial g_{2}\right)_{g}}{\left(\partial n / \partial g_{1}\right)_{g}}\left(\frac{\partial g_{2}}{\partial g_{1}}\right)_{\mu_{2}}\right]^{2}
$$

and the relation between $B$ and $B^{*}$ is

$$
B^{*}=B\left[1+\frac{\left(\partial n / \partial g_{2}\right)_{g}}{\left(\partial n / \partial g_{1}\right)_{g}}\left(\frac{\partial g_{2}}{\partial g_{1}}\right)_{\mu_{2}}\right]^{-2} .
$$

Relations equivalent to Eq. [26a] were already used by Stockmayer (5) and by Ooi ( 7 ) in the discussion of the adsorption from a mixed solvent to an uncharged polymer as measured by Ewart et al. (19). More recently 
Strauss and Wineman (20) used an equation equivalent to Eq. [24] for the determination of the molecular weight of polyphosphates. They determined a quantity analogous to $\left(\partial g_{2} / \partial g_{1}\right)_{\mu_{2}}$ from membrane equilibria.

\section{The Negative Adsorption of Salt}

The most obvious contribution to $\left(\partial g_{2} / \partial g_{1}\right)_{\mu_{2}}$ is caused by the electrical double layer. If we neglect any specific effects, the double layer is always a seat of negative adsorption of the supporting electrolytes. Assuming for simplicity this to be $\mathrm{NaCl}$, the concentrations of the ions in the double layer are given by

$$
\begin{aligned}
& c_{N a}=c_{2} \exp (-e \psi / k T) \\
& c_{c l}=c_{2} \exp (+e \psi / k T) .
\end{aligned}
$$

Here $e$ is the elementary charge, and $\psi$ is the potential in the double layer. It has been pointed out repeatedly (for example, Overbeek (21)) that according to Eq. [27] the excess of counterions and the deficit of co-ions are equal when the potential is low, but that at increasing potential the excess of counterions rises much faster than the deficit of co-ions. See Fig. 2.

The difference between the amounts of counterions and co-ions just compensates the particle charge ze. The negative adsorption of salt is equal to the deficit of co-ions and thus equal to $\alpha z$ molecules per particle, where $\alpha=1 / 2$ for low potentials and $\alpha<1 / 2$ for high potentials. Stigter (22), for example, has calculated $\alpha$ for spherical micelles of sodiumlaurylsulfate with a charge of about 100 and has found $\alpha \sim 0.085$.

We may thus write

$$
\left(\frac{\partial g_{2}}{\partial g_{1}}\right)_{\mu_{2}}=\frac{M_{2}}{M_{1}}\left(\frac{\partial c_{2}}{\partial c_{1}}\right)_{\mu_{2}}=-\frac{M_{2} z \alpha}{M_{1}}
$$
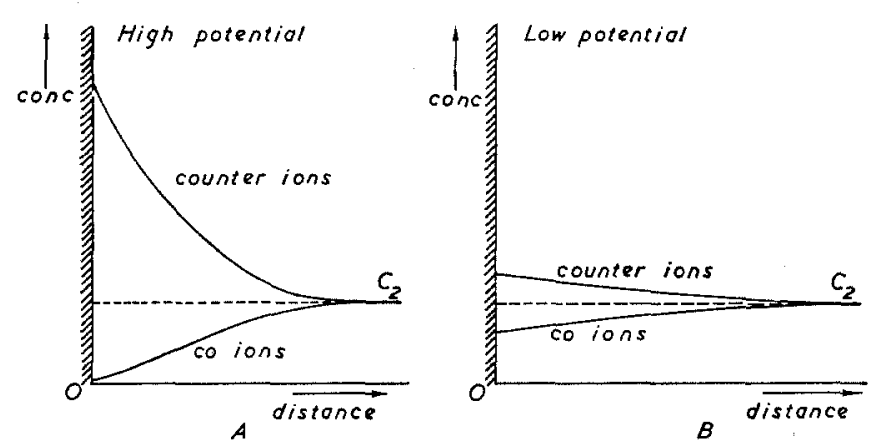

FIg. 2. Illustrating the distribution of ions in double layers. $A$ for high potentials; $B$ for low potentials. 
and

$$
M_{1}^{*}=M_{1}\left(1-\alpha z \frac{M_{2}}{M_{1}} \frac{\left(\partial n / \partial g_{2}\right)_{g}}{\left(\partial n / \partial g_{1}\right)_{g}}\right)^{2} .
$$

Mysels and co-worker $(9,10)$ have treated the light scattering by micelles of sodiumlaurylsulfate assuming incomplete dissociation (effective charge $\left.z_{e f f} . e: z e\right)$ combined with ideal behavior. They obtain an equation which can be written in a first approximation

$$
M_{1}^{*}=M_{1}\left(1-\frac{z_{\text {eff }}}{2} \frac{M_{2}\left(\partial n / \partial g_{2}\right)_{g}}{M_{1}\left(\partial n / \partial g_{1}\right)_{g}}\right)^{2} .
$$

Their $z_{\text {eff }}$. is therefore equal to $2 \alpha z$, twice the negative adsorption according to the above treatment. They find $z_{e f f} \simeq 15$, nearly identical with $2 \alpha z=17$, which follows from Stigter's work.

\section{EXPERIMENTS}

The theory developed above has been tested with solutions of polymethacrylic acid (PMA) in aqueous $\mathrm{HCl}$ and in various salt solutions. In $\mathrm{HCl}$ no double layer effects are to be expected, because the polymer is uncharged. In salt solutions, the polymer was half neutralized by addition of $\mathrm{NaOH}$, and charge effects are pronounced. As the structure of the polymer coil is mainly determined by the concentration and valence of the cations, while the optical effects are just as much determined by the anions, we have directed our attention in particular to variation of the anions.

\section{Preparation of the Polymer}

PMA was prepared aceording to the method of Arnold and Overbeek (23) with $\mathrm{H}_{2} \mathrm{O}_{2}$ and $\mathrm{Fe}^{3+}$-ions as the catalyst ( $50 \mathrm{~g}$. methacrylic acid, $67 \mathrm{ml} .0 .03 \mathrm{M} \mathrm{H}_{2} \mathrm{O}_{2}, 70 \mathrm{ml} .0 .03$ $M \mathrm{FeSO}_{4}$, and $500 \mathrm{ml} . \mathrm{H}_{2} \mathrm{O}$ ). The PMA thus obtained has not been fractionated sharply; care has been taken only to get rid of the highest and lowest molecular weights by precipitation with $\mathrm{HCl}$ from aqueous solutions. To remove the iron the PMA was dissolved in an $\mathrm{NaOH}$ solution and filtered. Colloidal $\mathrm{Fe}(\mathrm{OH})_{3}$, however, is still present after this procedure. Upon addition of acetone the $\mathrm{Fe}(\mathrm{OH})_{3}$ precipitates together with a small amount of polymer and then the supernatant polymer solution can be decanted. The alkaline-polymer solution was then neutralized with $\mathrm{HCl}$ and dialyzed against distilled $\mathrm{H}_{2} \mathrm{O}$; subsequently the solution was electrodialyzed against conductivity water during 5 days. The solution obtained in this way was diluted to about $2 \%$ as determined by titration with $\mathrm{NaOH}$. It still contained a trace (less than $0.005 \%$ calculated on the polymer) of iron.

\section{Refractive Index InCREment}

A known weight of the stock solution of PMA was half neutralized by $\mathrm{NaOH}$ and after adding a known weight of salt, was diluted with distilled water to $50 \mathrm{ml}$. The refractive index increment determined was sufficiently independent of the wavelength to allow determination with white light in a 
TABLE I

Refractive Index Increments of Salt and of PMA at $20^{\circ} \mathrm{C}$.

\begin{tabular}{llccc}
\hline \multicolumn{1}{c}{ Solvent } & $\begin{array}{c}M_{2}\left(\frac{\partial n}{\partial g_{2}}\right)_{g_{1}=0} \\
(m l . / m o l e)\end{array}$ & $\begin{array}{c}\left(\frac{\partial n}{\partial g_{1}}\right)_{g 2} \\
\left(m l^{2} / g .\right)\end{array}$ & $\begin{array}{c}\left(\frac{\partial n}{\partial g_{1}}\right)_{\mu_{2}} \\
(m l . / g .)\end{array}$ \\
\hline $0.045 \mathrm{M} \mathrm{HCl}$ & - & 0.156 & 0.162 \\
0.1 & $M \mathrm{NaF}$ & 5.5 & 0.229 & 0.219 \\
0.1 & $M \mathrm{NaCl}$ & 10.2 & - & 0.213 \\
0.1 & $M \mathrm{NaBr}$ & 13.4 & 0.234 & 0.209 \\
0.1 & $M \mathrm{NaI}$ & 21.2 & - & 0.197 \\
0.1 & $M \mathrm{Na} \mathrm{SO}_{4}$ & 21.3 & - & 0.210 \\
0.01 & $M\left(\mathrm{NH}_{4}\right)_{6} \mathrm{MO}_{7} \mathrm{O}_{24}$ & - & 0.227 & 0.169 \\
\hline
\end{tabular}

The polymer in the salt solutions is half neutralized; concentrations $g_{1}$ are expressed in gram PMA (acid form) per milliliter.

Rayleigh interferometer (fabricated by Zeiss). It was determined for constant salt concentration (usually $0.1 M$ ) and various PMA concentrations (maximum $0.15 \%$ ). The solutions were then dialyzed against the same salt concentration. After about 24 hours equilibrium was established; the polymer concentration had not changed materially. The refractive index increment (now at constant chemical potential) was again determined. The results are given in Table $\mathbf{I}$.

\section{SCATtering of Light}

Scattering of light at angles of 45,90 , and 135 degrees with the incident beam was measured with a Brice-Phoenix apparatus at $546 \mathrm{~m} \mu$. For each salt concentration a series of polymer concentrations (half neutralized with $\mathrm{NaOH}$ ) were investigated. With the $\mathrm{HCl}$ solution dissymmetry was nearly absent $\left(z=I_{45} / I_{135}=1.02 ; P_{90}^{-1}=1.014\right.$; for notation see Doty and Steiner (24)); in the salt solutions it was somewhat higher (average $z=1,1 ; P_{90}^{-1}=1.08$ ). The turbidity $\tau$ used in our equations has been calculated from the light scattered at $90^{\circ}$ by the equation

$$
\tau=\frac{I_{90} \circ r^{2}}{I_{\text {prim. }}} P_{90}^{-1} \frac{16 \pi}{3}
$$

where $r$ is the distance from the primary beam.

In Fig. 3 the values of $H g_{1} /\left(\tau-\tau^{*}\right)$ have been plotted against $g_{1}$, the PMA concentration. The refractive index increment in $H$ was taken at constant salt concentration. So the lines extrapolate to $1 / M^{*}$ rather than to $1 / M_{1}$ and have a slope $B^{*}$ rather than $B$. In all cases, except for $\mathrm{HCl}$ solutions, the average value 0.230 has been used for $\left(\partial n / \partial g_{1}\right)_{g_{2}}$.

The line for $\mathrm{HCl}$ is nearly horizontal, indicating a very small interaction between polymer particles. In salt solutions where the polymer is charged, 


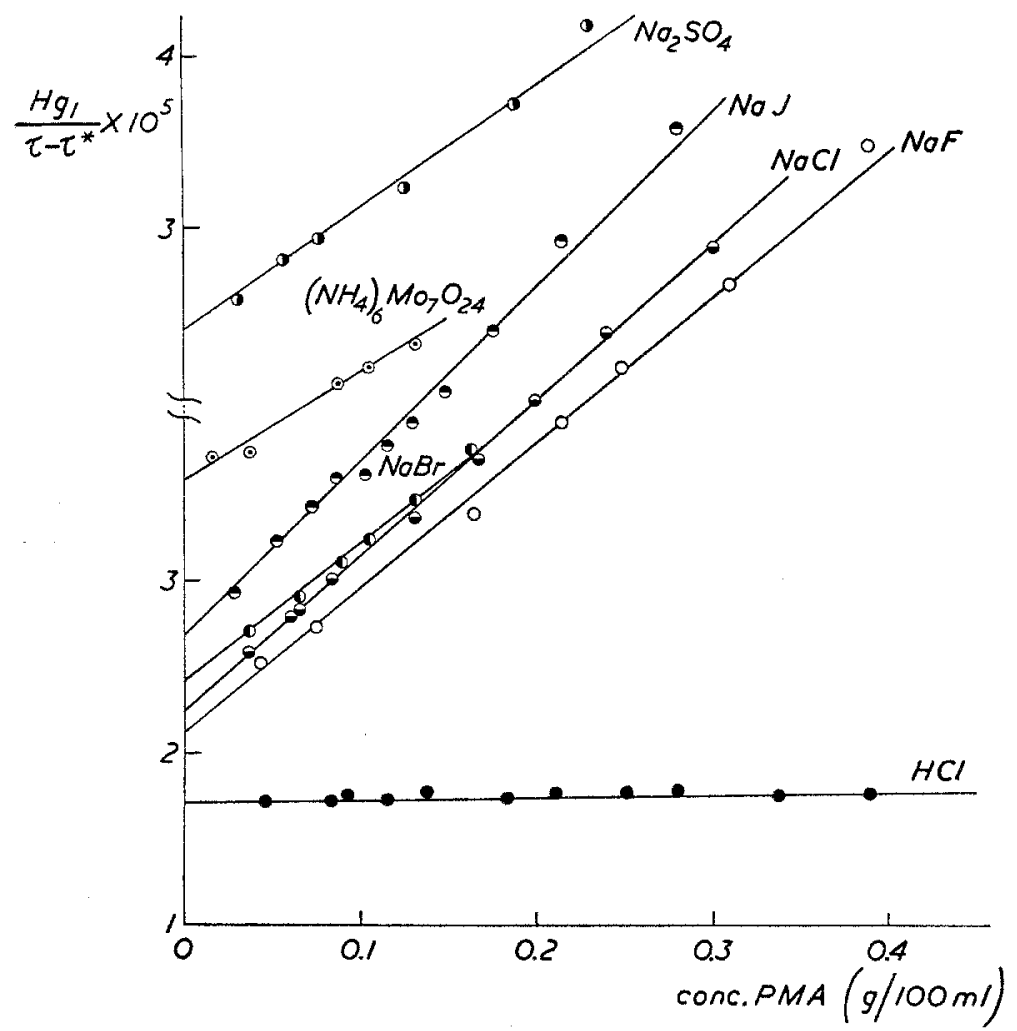

FIG. 3. $H g_{1} /\left(\tau-\tau^{*}\right)$ plotted against concentration PMA. The data for $\mathrm{Na}_{2} \mathrm{SO}_{4}$ have been shifted upwards for clarity.

TABLE II

Apparent and True Molecular Weight and Second Virial Coefficient of PMA

\begin{tabular}{llcccc}
\hline \multicolumn{1}{c}{ Solvent } & $M_{1}{ }^{*} \times 10^{-3}$ & $M_{1} \times 10^{-3}$ & $\begin{array}{c}B^{*} \times 10^{3} \\
(m l . / g .)\end{array}$ & $\begin{array}{c}B \times 10^{3} \\
(m l . / g .)\end{array}$ \\
\hline $0.045 \mathrm{M} \mathrm{HCl}$ & 58.0 & 53.8 & 0 & 0 \\
0.1 & $M \mathrm{NaF}$ & 46.7 & 51.5 & 4.12 & 3.74 \\
0.1 & $M \mathrm{NaCl}$ & 44.7 & 52.1 & 4.35 & 3.73 \\
0.1 & $M \mathrm{NaBr}$ & 41.2 & 50.0 & 4 & 3.3 \\
0.1 & $M \mathrm{NaI}$ & 37.2 & 50.7 & 5.00 & 3.66 \\
0.1 & $M \mathrm{Na}_{2} \mathrm{SO}_{4}$ & 41.5 & 49.8 & 3.42 & 2.85 \\
0.01 & $M\left(\mathrm{NH}_{4}\right)_{6} \mathrm{Mo}_{7} \mathrm{O}_{21}$ & 28 & 52 & 2.95 & 1.59 \\
\hline
\end{tabular}

the slope is pronounced, indicating strong repulsion between the particles. The cut-offs on the ordinate correspond to $1 / M_{1}^{*}$ (Eq. [26a]) and the slopes to $B^{*}$ (Eq. [26b]). They are different for each of the salts. If the correction factor $\left[1+\left(\partial g_{2} / \partial g_{1}\right)_{\mu_{2}}\left(\partial n / \partial g_{2}\right)_{g} /\left(\partial n / \partial g_{1}\right)_{g}\right]^{2}$ is applied, the true 
molecular weight $M_{1}$ found from the different salt solutions appears to be $51.000 \pm 1000$. The second virial coefficients, $B$, for the different halides are nearly identical, indicating that the interactions between pairs are also very similar. See Table II.

\section{Negative Adsorption and Extrapolation Procedure for the MoleCULAR Weight}

The difference between the refractive index increment at constant concentration and at constant chemical potential of the supporting electrolyte is directly connected with the (negative) adsorption of the salt to the polymer.

The salt adsorption in grams per gram of polymer, $\left(\partial g_{2} / \partial g_{1}\right)_{\mu_{2}}$ or in moles per equivalent weight of polymer $\partial\left(g_{2} / M_{2}\right) / \partial\left(g_{1} / E\right)_{\mu_{2}}$ can be determined from the following equation

$$
\begin{aligned}
\left(\partial n / \partial g_{1}\right)_{\mu_{2}}=\left(\partial n / \partial g_{1}\right)_{g}+\left(\partial n / \partial g_{2}\right)_{g}\left(\partial g_{2} / \partial g_{1}\right)_{\mu_{2}} & \\
& =\left(\partial n / \partial g_{1}\right)_{g}+\left(\frac{\partial n}{\partial g_{2}}\right)_{g} \frac{M_{2}}{E} \frac{\partial\left(g_{2} / M_{2}\right)}{\partial\left(g_{1} / E\right)},
\end{aligned}
$$

where $E$ is the equivalent weight of the polymer (half neutralized!, $E=172$ ) and $M_{2}$ is the formula weight of the salt. Some values of the negative adsorption can be calculated with the help of Table I, but they are not very accurate. Nevertheless it is obvious that all the salts are negatively adsorbed, $\left(\left(\partial n / \partial g_{1}\right)_{\mu_{2}}<\left(\partial n / \partial g_{1}\right)_{g_{2}}\right)$, in agreement with simple electrostatic theory. The slight positive adsorption of $\mathrm{HCl}$ must be due to a more specific effect.

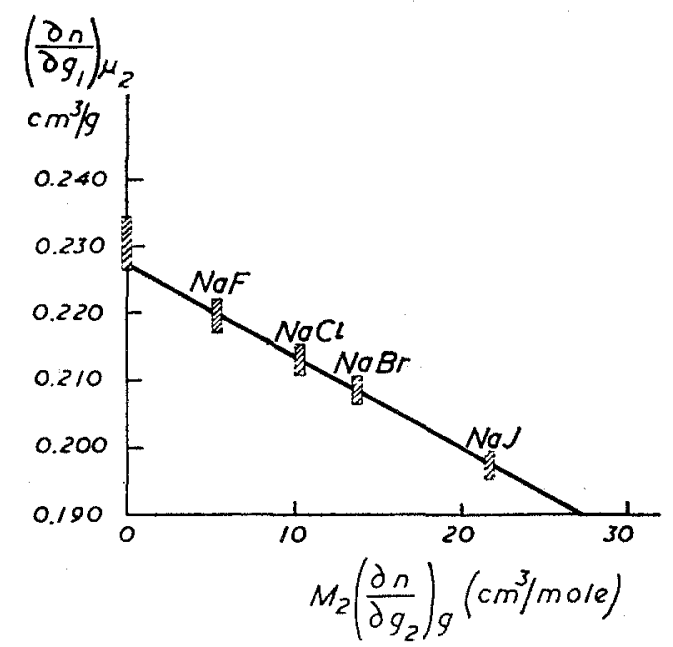

FIG. 4. Refractive index increment at constant chemical potential plotted against molar refractive index increment of salt. 


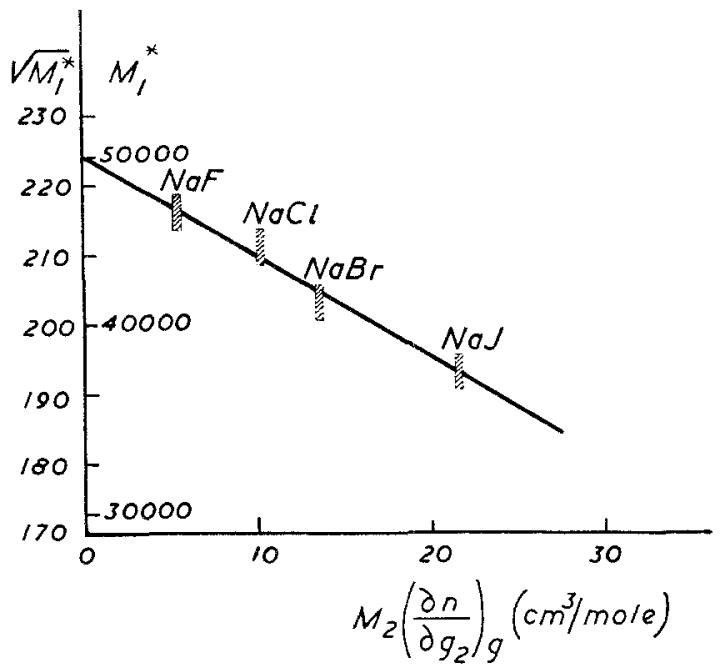

FIG. 5. Square root of the apparent molecular weight $M_{1}{ }^{*}$ plotted against molar ref ractive index increment of salt.

A more accurate value for the negative adsorption can be obtained on the assumption that it has the same vallue for the four halides. Then, if $\left(\partial n / \partial g_{1}\right)_{\mu_{2}}$ is plotted against $M_{2}\left(\partial n / \partial g_{2}\right)_{g}$, the slope of the straight line obtained gives the negative adsorption and the cut-off on the axis the value of $\left(\partial n / \partial g_{1}\right)_{g_{2}}$ as follows from Eq. [32]. From the plot in Fig. 4 the negative adsorption of the halides appears to be 0.24 mole/equivalent of polymer.

Similar use can be made of Eq. [26a]. By plotting the square root of the apparent molecular weight $\left(\sqrt{M^{*}}\right)$ against $M_{2}\left(\partial n / \partial g_{2}\right)_{g}$ (Fig. 5) a straight line is obtained.

From the cut-off the true molecular weight is found $(M=50000)$ and the slope gives the negative adsorption $\left(\partial\left(g_{2} / M_{2}\right) / \partial\left(g_{1} / E\right)\right)_{\mu_{2}}=0.25$ mole/equivalent), both in very good agreement with the values determined earlier. An attractive feature of this method is that it foregoes the use of the membrane equilibrium and the separate determination of the negative adsorption. It allows determination of the molecular weight and the negative adsorption from conventional light-scattering and refractive index increment measurements only.

\section{Appendix I}

Derivation of the Light-Scattering EQ. [12]

For the conversion of Eq. [8]

$$
\overline{\frac{\Delta n^{2}}{k T}}=\sum_{i, k \leq 0}^{m}\left(\partial n / \partial N_{i}\right)_{V T N}\left(\partial n / \partial N_{k}\right)_{V T N}\left(\partial N_{k} / \partial \mu_{i}\right)_{V T_{\mu} \mu}
$$


auxiliary equations for $\left(\partial n / \partial N_{i}\right)_{V T N}$ are first derived, in which the concentrations $c_{i}(i=1 \cdots m)$ and $P$ are considered as the independent variables instead of $N_{i}(i=0 \cdots m)$ and $V$ :

$$
\begin{aligned}
\left(\frac{\partial n}{\partial N_{i}}\right)_{V N} & =\left(\frac{\partial n}{\partial N_{i}}\right)_{P, N}+\left(\frac{\partial n}{\partial V}\right)_{N}\left(\frac{\partial V}{\partial P}\right)_{N}\left(\frac{\partial P}{\partial N_{i}}\right)_{V, N} \\
& =\left(\frac{\partial n}{\partial N_{i}}\right)_{P, N}-\left(\frac{\partial n}{\partial V}\right)_{N}\left(\frac{\partial V}{\partial N_{i}}\right)_{P, N}=\left(\frac{\partial n}{\partial N_{i}}\right)_{P, N}-n_{V} V_{i} \\
& =\sum_{k=1}^{m}\left(\frac{\partial n}{\partial c_{k}}\right)_{P, c}\left(\frac{\partial c_{k}}{\partial N_{i}}\right)_{P, N}-n_{V} V_{i} .
\end{aligned}
$$

With $c_{k}=N_{k} / V$ this is transformed into

$$
\begin{aligned}
\left(\frac{\partial n}{\partial N_{i}}\right)_{V N} & =\left(\frac{\partial n}{\partial c_{i}}\right)_{P, c} \frac{1}{V}-\sum_{k=1}^{m}\left(\frac{\partial n}{\partial c_{k}}\right)_{P, c} \frac{c_{k}}{V}\left(\frac{\partial V}{\partial N_{i}}\right)_{P, N}-n_{V} V_{i} \\
& =\left[\gamma_{i}-V_{i}\left(n_{V} V+\phi\right)\right] / V .
\end{aligned}
$$

It should be remembered that $\gamma_{i}$ does not exist for $i=0$. Inserting the expressions [A1] into Eq. [8] leads to

$$
\begin{aligned}
\frac{\overline{\Delta n^{2}}}{k T}=\frac{1}{V^{2}} \sum_{i, k=1}^{m} \gamma_{i} & \gamma_{k}\left(\partial N_{k} / \partial \mu_{i}\right)_{V, \mu} \\
& -\frac{2\left(n_{V} V+\phi\right)}{V^{2}} \sum_{\substack{i=1 \\
k=0}}^{m} \gamma_{i} V_{k}\left(\partial N_{k} / \partial \mu_{i}\right)_{V, \mu} \\
& +\frac{\left(n_{V} V+\phi\right)^{2}}{V^{2}} \sum_{i, k=0}^{m} V_{i} V_{k}\left(\frac{\partial N_{k}}{\partial \mu_{i}}\right)_{V, \mu}
\end{aligned}
$$

Using the Gibbs-Duhem relation and differentiating with respect to $N_{k}$ at constant $V$ and $N_{0} \cdots N_{k-1}, N_{k+1} \cdots N_{m}$ we obtain

$$
V\left(\partial P / \partial N_{k}\right)_{V N}=\sum_{l=0}^{m} N_{l}\left(\partial \mu_{l} / \partial N_{k}\right)_{V, N} .
$$

Substituting

$$
\left(\partial P / \partial N_{k}\right)_{V, N}\left(\partial N_{k} / \partial V\right)_{P, N}(\partial V / \partial P)_{N}=-1
$$

and

$$
\kappa=-\frac{1}{V}\left(\frac{\partial V}{\partial P}\right)_{N}
$$

we find

$$
V_{k}=\kappa \sum_{l=0}^{m} N_{l}\left(\partial \mu_{l} / \partial N_{k}\right)_{V, N}
$$


Further,

$$
\sum_{k=0}^{m}\left(\partial \mu_{l} / \partial N_{k}\right)_{V, N}\left(\partial N_{k} / \partial \mu_{i}\right)_{\mu, V}=\left(\partial \mu_{l} / \partial \mu_{i}\right)_{V, \mu}=\delta_{i l}
$$

where $\delta_{i l}=0$ if $i \neq l$ and $\delta_{i l}=1$ if $i=l$. Inserting [A4] into [A2] and carrying out the summation over $k$ leads to

$$
\begin{aligned}
\frac{V^{2} \overline{\Delta n^{2}}}{k T}=\sum_{i, k=1}^{m} \gamma_{i} \gamma_{k}\left(\partial N_{k} / \partial \mu_{i}\right)_{V, \mu}-2\left(n_{V} V+\phi\right)_{\kappa} \sum_{\substack{i=1 \\
l=0}}^{m} \gamma_{i} N_{l} \delta_{i l} \\
+\left(n_{V} V+\phi\right)^{2} \kappa \sum_{\substack{i=0 \\
l=0}}^{m} V_{i} N_{l} \delta_{i l} .
\end{aligned}
$$

With $\sum_{i=1}^{m} \gamma_{i} N_{i}=V \phi$ and $\sum_{i=0}^{m} V_{i} N_{i}=V$ this expression reduces to

$$
\frac{V \overline{\Delta n^{2}}}{k T}=\left(V^{2} n_{V}{ }^{2}-\phi^{2}\right) \kappa+\frac{1}{V} \sum_{i, k=1}^{m} \gamma_{i} \gamma_{k}\left(\partial N_{k} / \partial \mu_{i}\right)_{V, \mu} .
$$

It is convenient to transform the differentiation with $\mu_{1}=$ constant in Eq. [A6] into one with $N_{1}=$ constant but keeping to $\mu=$ constant for the other components. This will be indicated by the suffix $\mu_{s}$. We find

$$
\begin{aligned}
& \text { for } i, k \neq 1 \quad\left(\partial N_{k} / \partial \mu_{i}\right)_{V, \mu}=\left(\partial N_{k} / \partial \mu_{i}\right)_{V, \mu_{s}, N_{1}} \\
& +\left(\partial N_{k} / \partial N_{1}\right)_{V, \mu_{\delta}}\left(\partial N_{1} / \partial \mu_{i}\right)_{V, \mu} \\
& \text { for } i=1 \quad \text { or } \quad k=1 \quad\left(\partial N_{k} / \partial \mu_{i}\right)_{Y, \mu} \\
& =\left(\partial N_{k} / \partial N_{1}\right)_{V, \mu_{s}}\left(\partial N_{1} / \partial \mu_{i}\right)_{\nabla, \mu} .
\end{aligned}
$$

Applying Eq. [9] leads to

$$
\left(\partial N_{1} / \partial \mu_{i}\right)_{V, \mu}=\left(\partial N_{i} / \partial \mu_{1}\right)_{V, \mu_{s}}=\frac{\left(\partial N_{i} / \partial N_{1}\right)_{V, \mu_{s}}}{\left(\partial \mu_{1} / \partial N_{1}\right)_{V, \mu_{s}}}
$$

From the Gibbs-Duhem relation we find

$$
N_{1}\left(\partial \mu_{1} / \partial N_{1}\right)_{V, \mu_{s}}=V\left(\partial P / \partial N_{1}\right)_{V, \mu_{s}} .
$$

Combining Eqs. [A6], $[A 7],[A 8]$, and [A9] finally gives

$$
\begin{aligned}
\frac{V \overline{\Delta n^{2}}}{k T}=\left(V^{2} n_{V}{ }^{2}-\phi^{2}\right)_{\kappa}+\frac{1}{V} \sum_{i, k=2}^{m} \gamma_{i} \gamma_{k}\left(\partial N_{k} / \partial \mu_{i}\right)_{V, \mu_{s}, N_{1}} \\
+\frac{\sum_{i, k=1}^{m} \gamma_{i} \gamma_{k}\left(\frac{\partial N_{k}}{\partial N_{1}}\right)_{V, \mu_{s}}\left(\frac{\partial N_{i}}{\partial N_{I}}\right)_{V, \mu_{s}} N_{1}}{V^{2}\left(\partial P / \partial N_{1}\right)_{V, \mu_{s}}}
\end{aligned}
$$

which gives Eq. [12] after conversion to concentrations $c_{i}=N_{i} / V$, making use of Eqs. [5] and [13], and writing II for $P$. Indeed $V\left(\partial P / \partial N_{1}\right)_{V, \mu_{s}}$ or 
$\left(\partial P / \partial c_{1}\right)_{\mu_{s}}$ represents the change in pressure of the colloidal solution, with the colloid concentration at constant chemical potentials of all other components, and this corresponds exactly with the change in the Donnan pressure of the colloidal solution.

\section{APPENDIX II}

Estimation of the Difference Between the Turbidity Contributed BY the Low Molecular Weight Compounds in the

Colloid-Containing aNd in the Colloid-Free Solution

The difference between $\tau^{*} / A$ (see Eq. [19]) and the first and third term on the right-hand side of Eq. [12]

$$
\frac{\tau^{0 *}}{A}=\left[V^{2} n_{V}^{2}-\phi^{2}\right] \kappa+\sum_{i, k=2}^{m} \gamma_{i} \gamma_{k}\left(\partial c_{i} / \partial \mu_{k}\right)_{\mu_{s^{\prime}, c_{1}}}
$$

is caused by the presence of the colloid material with the concentration $c_{1}$, and is in first approximation proportional to $c_{1}$. The proportionality constant can be found by differentiating $\tau^{0 *}$ with respect to $c_{1}$ at constant $\mu_{s}$. It should then be compared to the proportionality constant of the main term

$$
\left[\sum_{i=1}^{m} \gamma_{i}\left(\partial c_{i} / \partial c_{1}\right)_{\mu_{s}}\right]^{2} /\left(\partial I / \partial c_{1}\right)_{\mu_{s}} .
$$

Writing $R$ for the ratio of $\left(\partial\left(\tau^{0 *} / A\right) / \partial c_{1}\right)$ to $[B 2]$ and introducing concentrations by weight

$$
g_{i}=c_{i} M_{i} / N_{\mathbf{A v}},
$$

where $M_{i}$ is the molecular weight of component $i$, we find

$$
R=\frac{\frac{N_{A v}}{M_{1}} \frac{\partial}{\partial g_{1}}\left\{\left[V^{2}\left(\frac{\partial n}{\partial V}\right)_{N}^{2}-\left(\sum_{i=1}^{m}\left(\frac{\partial n}{\partial g_{i}}\right)_{P_{g}} g_{i}\right)^{2}\right]_{\kappa}\right.}{\left.+\sum_{i, k=2}^{m}\left(\frac{\partial n}{\partial g_{i}}\right)_{P_{\theta}}\left(\frac{\partial n}{\partial g_{k}}\right)_{P_{g}}\left(\frac{\partial g_{i}}{\partial \mu_{k}}\right)_{\mu_{s}^{\prime} g_{1}} \frac{M_{k}}{N_{A v}}\right\}} .
$$

A reasonable estimate of $R$ can be obtained by the following simplifications and substitutions. All $\left(\partial n / \partial g_{i}\right)$ are taken as equal, independent of $g_{1}$, and about equal to $+0.3 ; V(d n / d V)$ is of the same order, but negative: $\partial / \partial g_{1}[V(d n / d V)]$ can be estimated at about $-0.1 ; \sum_{1}^{m_{2}}\left(\partial g_{i} / \partial g_{1}\right)$, which includes the term $\left(\partial g_{1} / \partial g_{1}\right)=1$ and the (negative) adsorptions $\left(\partial g_{i} / \partial g_{1}\right)_{i \neq 1}$, can be estimated at 0.5 or higher; $\partial \Pi / \partial c_{1}=k T ; \kappa$ is equal to $5 \times 10^{-11} \mathrm{ergs} / \mathrm{cm}^{3}{ }^{3}$ (for water) and $\frac{\partial \kappa}{\partial g_{1}}$ may vary between $-5 \times 10^{-11}$ and 
$+5 \times 10^{-11}$. The term $\partial / \partial g_{1} \sum_{i, k=2}^{m}\left(\partial g_{i} / \partial \mu_{k}\right)_{\mu_{s, g}^{\prime}}$ can be transformed as follows.

Neglecting activity corrections we put

$$
\mu_{k}=\mu_{k}^{0}+\nu_{k} k T \ln g_{k}^{0},
$$

where $\nu_{k}$ is a small integer and $g_{k}{ }^{0}$ is the weight concentration in the equilibrium solution at $g_{1}=0$. Now

$$
g_{i}=g_{i}^{0}+\beta_{i} g_{1},
$$

where $\beta_{i}$ depends on all $g_{i}^{0}$ 's. In the more simple cases $\sum_{i} \beta_{i}$ will be a function of $\sum_{2}^{m} g_{i}^{0}$. At high electrolyte concentration it will reach a limit. At low electrolyte concentration it varies as (ionic $\operatorname{strength}^{1 / 2}$ or as $\left(\sum_{2}^{m} g_{i}^{0}\right)^{1 / 2}$. With these assumptions the actual transformation is simple.

$$
\begin{aligned}
\frac{\partial}{\partial g_{1}} \sum_{i, k=2}^{m}\left(\partial g_{i} / \partial \mu_{k}\right)_{\mu_{s, g}^{\prime}} & =\sum \partial^{2}\left(g_{i}{ }^{0}+\beta_{i} g_{1}\right) / \nu_{k} k T \partial g_{1} \partial \ln g_{k}{ }^{0} \\
= & \frac{1}{\nu k T} \sum_{k} \frac{\sum \beta_{i}}{\partial \ln g_{k}{ }^{0}}=\frac{1}{\nu k T} \frac{\partial \sum \beta_{i}}{\partial \ln \sum g_{k}{ }^{0}}=\frac{1 / 2 \sum \beta_{i}}{\nu k T} .
\end{aligned}
$$

Here $\nu$ is a weighted average between the $\nu_{k}$ 's. Taking this as equal to 2 and taking -0.5 for $\sum \beta_{i}$ (cf. the value chosen for $\sum\left(\partial g_{i} / \partial g_{1}\right)$ ), $R$ finally becomes

$$
R=\frac{4 R T}{M_{1}}\left(+3 \times 10^{-11} \pm 5 \times 10^{-11}-\frac{\overline{M_{k}}}{8 R T}\right) .
$$

With $R T=2.5 \times 10^{10}$ this reduces to

$$
R=+\frac{3}{M_{1}} \pm \frac{5}{M_{1}}-\frac{\overline{M_{k}}}{2 M_{1}} .
$$

Therefore the absolute error introduced is at most of the order of the average molecular weight of the low molecular solutes and probably never less than 5 or 10 units in the molecular weight.

Finally, we remark that a very similar, if not identical value for $R$ is found, if the differentiation of $[B 1]$ is not carried out at constant chemical potential, but at constant concentrations of the low molecular weight compounds.

\section{References}

1. Deвye, P., J. Phys. \& Colloid Chem. 51, 18 (1947).

2. Zernike, F., Arch. néerl. sci. IIIA, 74 (1918); Dissertation, Amsterdam, 1915.

3. Brinkman, H. C., and Hermans, J. J., J. Chem. Phys. 17, 574 (1949).

4. Kirkwood, J. G., And Goldberg, R. J., J. Chem. Phys. 18, 54 (1950).

5. Stockmayer, W. H., J. Chem. Phys. 18, 58 (1950).

6. ShogenJi, H., Busseiron Kenkyu 62, 1 (1953).

7. OoI, T., J. Polymer Sci. 28, 459 (1958). 
8. Casassa, E. F., and Eisenberg, H., J. Phys. Chem. 64, 753 (1960); ibid. 65, 427 (1961).

9. Mrsels, K. J., J. Colloid Sci. 10, 507 (1955).

10. Princen, L. H., ANd Mrsels, K. J., J. Colloid Sci. 12, 594 (1957).

11. Prins, W., and Hermans, J. J., Koninkl. Ned. Akad. Wetenschap. Proc. B59, 162 (1956).

12. VRIJ, A., Dissertation, Utrecht, Holland, 1959.

13. Stigter, D., J. Phys. Chem. 64, 842 (1960).

14. Doty, P., And Steiner, R. F., J. Chem. Phys. 20, 85 (1952).

15. Hermans, J. J., Rec. trav. chim. 68, 859 (1949); Proc. Intern. Colloq. Macromolecules, Amsterdam, 1950, p. 238.

16. See, for example, T. L. Hill, "Statistical Mechanics," p. 114. McGraw-Hill, New York, 1956.

17. Onsager, L., Ann. N. Y. Acad. Sci. 51, 627 (1949).

18. Hill, T. L., "Ion Transport across Membranes," p. 198. Academic Press, New York, 1954.

19. Ewart, R. H., Roe, C. P., Debye, P., and McCartney, J. R., J. Chem. Phys. 14, 687 (1946).

20. Strauss, U. P., and Wineman, P. L., J.Am. Chem. Soc. 80, 2366 (1958).

21. Overbeek, J. Th. G., "The Donnan equilibrium," Progr. Biophys. Biophys. Chem. 6, 66 (1956).

22. STIGTER, D., Rec. trav. chim. 73, 593 (1954).

23. Arnold, R., and Overbeek, J. TH. G., Rec. trav. chim. 69, 192 (1950).

24. Doty, P., And Steiner, R. F., J. Chem. Phys. 18, 1211 (1950). 